\title{
Aberrant Expression of HOXA5 and HOXA9 in AML
}

\author{
Peng Zhao ${ }^{1 \star}$, Li Tan ${ }^{2 \&}$, Jian Ruan ${ }^{3}$, Xiao-Ping Wei' ${ }^{2}$, Yi Zheng ${ }^{1}$, Li-Xia Zheng ${ }^{2}$, \\ Wei-Qin Jiang ${ }^{1}$, Wei-Jia Fang ${ }^{1 *}$
}

\begin{abstract}
Background: Aberrant expression of HOX gene expression has been observed in cancer. The purpose of this study was to investigate the alteration of HOXA5 and HOXA9 expression and their clinical significance in acute meloid leukemia (AML). Materials and Methods: The expression of HOXA5 and HOXA9 genes of bone marrow samples from 75 newly diagnosed AML patients and 22 healthy controls for comparison were examined by Realtime quantitative PCR (RQ-PCR) assay. Statistical analysis was conducted to evaluate HOXA5 and HOXA9 expression as possible biomarkers for AML. Results: The results showed that the complete remission rate $(52.6 \%)$ of the patients who highly expressed HOXA5 and HOXA9 was significantly lower than that $(88.9 \%)$ in patients who lowly express the genes $(P=0.015)$. Spearmann correlation coefficients indicated that the expression levels for HOXA5 and HOXA9 genes were highly interrelated $(r=0.657, P<0.001)$. Meanwhile, we detected significant correlations between HOXA9 expression and age in this limited set of patients $(P=0.009)$. Conclusions: The results suggest a prognostic impact of increased expression of HOXA5 and HOXA9 in AML patients.
\end{abstract}

Keywords: Acute myeloid leukemia - HOXA5 - HOXA9

Asian Pac J Cancer Prev, 16 (9), 3941-3944

\section{Introduction}

Homeobox (HOX) genes are members of a transcription factor family and play a crucial role in embryonic development and in the control of differentiation of adult hematopoietic cells (Cillo et al., 2001; Abramovich and Humphries, 2005; Spencer et al., 2015). Homeobox genes encode the class of transcription factors in vertebrates and are found in clusters called A, B, C, and D on four separate chromosomes. The HOXA genes are associated not only with the development of hematologic malignancies but also with the prognosis of these conditions. Some recent studies have shown that increased expression of HOXA genes is correlated to the cytogenetic findings associated with poor prognosis in AML and mixed lineage leukemia (Drabkin et al., 2002; Golub et al., 1999). It has been reported that HOXA5 and HOXA9 are relevant to the development of myelomonocytes (van Oostveen et al., 1999; Cooks et al., 1999; Fuller et al., 1999). The abnormal expression of HOXA9 was reported to affect the proliferation, differentiation, and apoptosis of hemopoietic cells (Golub et al., 1999), or even lead to leukemia (Nakamura et al., 1996; Borrow et al., 1996). In the present study, we sought to further investigate the HOXA5 and HOXA9 gene expression in 75 patients with acute myeloid leukemia (AML) to determine the relationship between HOXA5 and HOXA9 gene expression and AML pathogenesis and prognosis. Here, we show that HOXA5 and HOXA9 mRNA were highly expressed in 38/75 $(50.7 \%)$ AML patients. Spearmann correlation coefficients indicated that the expression levels for the two genes were highly interrelated. Importantly, the complete remission rate in these patients was lower than that of HOXA5 and HOXA9 non-expressors following the chemotherapy.

\section{Materials and Methods}

\section{Patients' samples}

This study included 75 patients who had a diagnosis of de novo AML at the First Affiliated Hospital of Guangzhou Medical University. The diagnosis and classification of AML patients were based on FrenchAmerica-British (FAB) and World Health Organization (WHO) criteria (Sabattini et al., 2010; Bennett et al., 1985). Treatment protocol was described as reported previously ( $\mathrm{Li}$ et al., 2013). Written informed consent was obtained from all patients. The study was approved by the Institutional Review Board of the First Affiliated Hospital of Guangzhou Medical University. The main clinical and laboratory features of the patient cohort were summarized in Table1. A total of 22 healthy donors were collected as controls.

\section{Cytogenetic analysis}

Conventional cytogenetic analysis was performed in the cytogenetics laboratory of the First Affiliated Hospital

${ }^{1}$ Department of Medical Oncology, The First Affiliated Hospital, School of Medicine, Zhejiang University, Hangzhou, ${ }^{2}$ Department of Hematology, the First Affiliated Hospital of Guangzhou Medical University, ${ }^{3}$ Cancer Center, Traditional Chinese Medicine-Integrated Hospital, Southern Medical University, Guangzhou, China ${ }^{\star}$ Equal contributors *For correspondence: weijiafang@zju.edu.cn 
of Guangzhou Medical University. Chromosomes were prepared routinely by the direct method or $24 \mathrm{~h}$ short-term culture of bone marrow cells. Karyotypes were analyzed on R-banded metaphases. Chromosome abnormalities were described according to the International System for Human Cytogenetic Nomenclature (Gonzalez et al., 2006).

RNA isolation, reverse transcription and real-time quantitative PCR

Mononuclear cells from bone marrows of AML patients at initial diagnosis and healthy donors were enriched by Ficoll-Hypaque gradient. Total RNA was isolated using Trizol reagent (Invitrogen, Carlsbad, CA, USA) following the manufacturer's instructions. cDNA was synthesized using two micrograms of total RNA, dNTPs, RNase inhibitor (RNAsin), MMLV reverse transcriptase (MBI Fermentas, Hanover, MD, USA), and random hexamers. The system of reverse transcription was incubated for $10 \mathrm{~min}$ at $25^{\circ} \mathrm{C}, 60 \mathrm{~min}$ at $42^{\circ} \mathrm{C}$, and then stored at $-20^{\circ} \mathrm{C}$. Real-time quantitative PCR (RQ-PCR) assay was performed for each sample in duplicate in ainal reaction volume of $20 \mu \mathrm{L}$. HOXA5 was amplified using the primers 5'GCGCAAGCTGCACATAAGTCA3' (forward) and 5'TGTCTCTCGGAGAGGCAAAGA3' (reverse) with expected products of 174bp. HOXA9 was amplified using the primers 5' ATCGATCCCAATAACCCAGCA3' (forward) and 5'TGGTGTTTTGTATAGGGGCAC3' (reverse) with expected products of $80 \mathrm{bp}$. Reaction system consisted of 20ng of cDNA, $0.8 \mu \mathrm{M}$ of primers, $10 \mu \mathrm{M}$ AceQTMqPCR SYBR Green Master Mix (Vazyme Biotech Co, Piscataway, NJ, USA), and $0.4 \mu \mathrm{M}$ ROX Reference Dye1 (Invitrogen). Reaction conditions were as follows: initial denaturation at $95^{\circ} \mathrm{C}$ for 5 minutes, 40 cycles of denaturation at $95^{\circ} \mathrm{C}$ for 15 seconds, and annealing and elongation at $58^{\circ} \mathrm{C}$ for 1 minute. Reverse transcription was performed in triplicate. The amount of target was calculated by the $2^{-\Delta \Delta \mathrm{Ct}}$ method (Arocho et al., 2006).

\section{Statistical analysis}

Statistical analysis was performed using SPSS 19.0 SPSS, Armonk, NY). Pearson $\chi^{2}$ analysis or Fisher exact test was employed to compare the difference of categorical variables. Mann-Whitney's U-test was used to compare the difference of continuous variables. Receiver operating characteristic curve (ROC) were used to set cut-off value of HOXA5 and HOXA9 expression in AML patients. Spearman rank correlation analysis was used to analyze the correlation of HOXA5 expression with expression of HOXA9. For all analyses, two-tailed p-values of 0.05 or less were determined statistically significant.

\section{Results}

HOXA5 and HOXA9 Gene Expression in de novo AML and Healthy Individuals

We evaluated the level of HOXA5 and HOXA9 expression in AML and healthy controls. The relative expression levels of HOXA5 and HOXA9 mRNA levels were significantly overexpressed in 75 de novo AML patients in comparison with the controls $(P<0.05)$
(Figure1). The expression of HOXA5 and HOXA9 was significantly higher in AML patients compared with normal subject.

\section{Correlation between HOXA5 and HOXA9 expression and clinical characteristics}

Overall, we studied 75 AML patients aged between 16 and 79 years (median 45 years) in this study (Table 1). AML subtypes, according to the French-AmericanBritish classification, were M2 ( $\mathrm{n}=28,37.3 \%), \mathrm{M} 3(\mathrm{n}=20$, $27 \%)$ non M2 and M3 ( $n=26,34.7 \%)$. Flow cytometric analysis of bone marrow cells revealed (2-93.5) \% (average $62.3 \%$ ) immature cells in the patients.

The relative expression levels of the HOXA5 and HOXA9 gene in 75 de novo AML patients according to the various clinical and pathological parameters are shown in Table1. Expression analysis regarding to the FAB type showed significant overexpression of HOXA9 in non M2 and M3 types $(P<0.05)$, low expression of HOXA9 were found in patients with M2, M3 and younger patients, especially in AML patients with $\mathrm{t}(8 ; 21)(\mathrm{q} 22 ; \mathrm{q} 22)$ and $\mathrm{t}$ $(15 ; 17)(\mathrm{q} 22 ; \mathrm{q} 21)$.

At the set cut-off value of 0.000102 and 0.00212 according to ROC curves, this cohort of AML patients was divided into four groups: high HOXA5 expression $(\geq 0.000102)$ and low HOXA5 expression $(<0.000102)$; high HOXA9 expression $(\geq 0.00212)$ and low HOXA9 expression $(<0.00212)$. There were no significant differences between low HOXA5 and high HOXA5 expressing patients with respect to age, white blood cells (WBCs), the percentage of blasts in bone marrow, FAB and karyotypes (Table1). Significant difference was seen between low HOXA5 and HOXA9 and patients with high HOXA5 and HOXA9 in the rates of complete remission after induction therapy $(P=0.015)$. However, significant differences can be seen between low HOXA9 and high HOXA9 expressing patients with respect to age $(P=0.009)$ and $\operatorname{FAB}(P=0.034)$ (Table 1$)$.

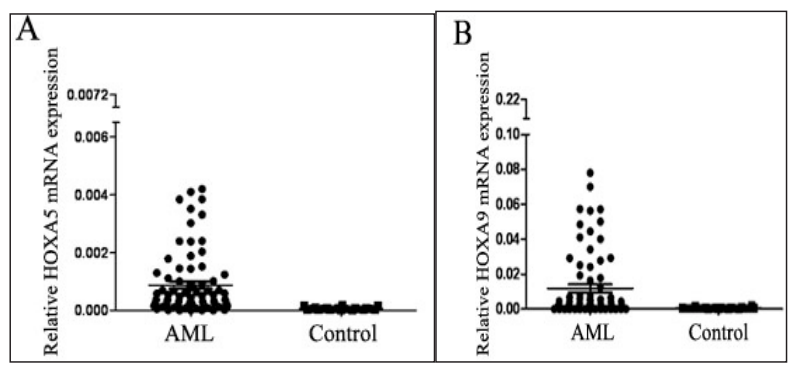

Figure 1. Relative Expression levels of HOXA5 and HOXA9 mRNA in AML and Control

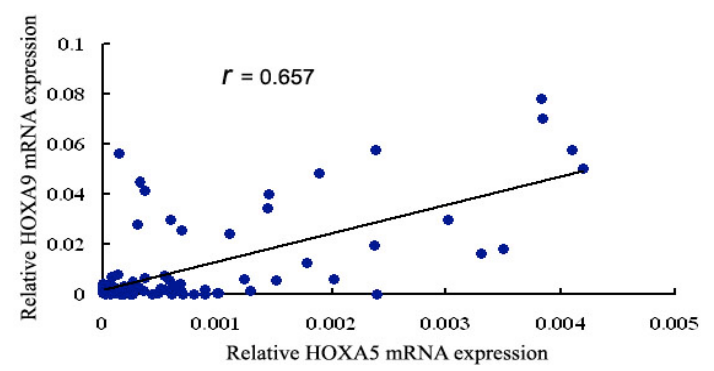

Figure 2. Results of qRT-PCR Performed for $\mathbf{7 5}$ Newly Diagnosed AML Patients, Expression of HOXA5 Correlated with HOXA9 $(r=0.657, P<0.001)$ 
Table 1. Relationship between Relation Gene Expression Level and Standard Clinical and Biological Factors

\begin{tabular}{|c|c|c|c|c|c|c|c|}
\hline \multirow[t]{2}{*}{ Grouping variable } & & \multicolumn{3}{|c|}{ HOXA5 n (\%) } & \multicolumn{3}{|c|}{ HOXA9 n $(\%)$} \\
\hline & & Low $(n=37)$ & $\operatorname{High}(\mathrm{n}=38)$ & $p$ value & Low $(n=37)$ & High $(n=38)$ & $p$ value \\
\hline \multirow[t]{2}{*}{ Age (years) } & $\leq 60$ & $33(88.9 \%)$ & $24(63.2 \%)$ & 0.070 & $35(94.4 \%)$ & $22(57.9 \%)$ & 0.009 \\
\hline & $>60$ & $4(11.1 \%)$ & $14(36.8 \%)$ & & $2(5.56 \%)$ & $16(42.1 \%)$ & \\
\hline \multirow[t]{2}{*}{ WBC } & $<30 \times 10^{9} / \mathrm{L}$ & $29(77.7 \%)$ & $32(84.2 \%)$ & 0.629 & $31(83.3 \%)$ & $30(78.9 \%)$ & 0.934 \\
\hline & $\geq 30 \times 10^{9} / \mathrm{L}$ & $8(22.2 \%)$ & $6(15.8 \%)$ & & $6(16.7 \%)$ & $8(21 \%)$ & \\
\hline \multirow[t]{2}{*}{ Blasts in BM } & $\leq 70 \%$ & $21(55.6 \%)$ & $20(52.8 \%)$ & 0.641 & $16(44.4 \%)$ & $22(57.9 \%)$ & 0.260 \\
\hline & $>70 \%$ & $16(44.4 \%)$ & $18(47.4 \%)$ & & $21(55.6 \%)$ & $16(42.1 \%)$ & \\
\hline \multirow[t]{2}{*}{ Karyotype } & Normal & $8(22.2 \%)$ & $16(42.1 \%)$ & 0.309 & $16(44.4 \%)$ & $16(42.1 \%)$ & 0.393 \\
\hline & Abnormal & $23(62.5 \%)$ & $18(47.4 \%)$ & & $16(44.4 \%)$ & $20(52.6 \%)$ & \\
\hline \multirow[t]{2}{*}{ Karyotype t $(8 ; 21)(\mathrm{q} 22 ; \mathrm{q} 22)$} & Absent & $29(77.8 \%)$ & $34(88.9 \%)$ & 0.348 & $27(72.2 \%)$ & $36(94.7 \%)$ & 0.074 \\
\hline & Present & $8(22.2 \%)$ & $4(10.5 \%)$ & & $10(27.8 \%)$ & $2(5.3 \%)$ & \\
\hline \multirow[t]{3}{*}{ FAB } & M2 & $16(44.4 \%)$ & $12(31.6 \%)$ & 0.186 & $19(51.3 \%)$ & $10(26.3 \%)$ & 0.034 \\
\hline & M3 & $12(33.3 \%)$ & $8(21 \%)$ & & $12(33.3 \%)$ & $8(21 \%)$ & \\
\hline & Other & $8(22.2 \%)$ & $18(47.4 \%)$ & & $6(16.7 \%)$ & $20(52.6 \%)$ & \\
\hline CR (-) & & $33(88.9 \%)$ & $20(52.6 \%)$ & 0.015 & $33(88.9 \%)$ & $20(52.6 \%)$ & 0.015 \\
\hline
\end{tabular}

Relation between HOXA5 and HOXA9 expression

Spearmann correlation coefficients indicated that the HOXA5 expression correlated with expression of HOXA9 ( $\mathrm{r}=0.657, P<0.001 ;$ Figure 2$)$.

\section{Discussion}

Acute myeloid leukemia (AML), the most common type of hematological malignancies in adults, is characterized by differentiation arrest and accumulation of myeloid blasts in bone marrow that leads to the insufficiency of normal hematopoiesis (Estey and Döhner, 2006; Shahab et al., 2013). Some markers have been shown to predict the clinical outcome and provide potential targets for molecular therapies, which are $<30 \%$ of de novo AML (Cancer Genome Atlas Research Network, 2013; Hou et al., 2014; kumar, 2014). Therefore, the molecular pathogenesis of AML has not yet been completely defined, new molecular markers are warranted to identify those who are at the risk of poor outcome and to optimize treatment strategies in patients with a normal karyotype and no date. In cancer, deregulation of HOX gene expression and HOX alterations have been most convincingly demonstrated in leukemia (Nakamura al., 1996; Nakamura al., 2009). In this report, we have used real-time RT-PCR to study HOXA5 and HOXA9 expression in defined cases of AML with clinical followup. The loss of HOXA5 function limits leukaemia associated with specific chromosomal translocations, which is a key regulator of myeloid differentiation (Boucherat al., 2009). Overexpression of HOXA5 in CD34+progenitor cells induced increased levels of granulocytic/monocytic differentiation and inhibited erythroid/megakaryocytic differentiation. Inhibition of HOXA5 inhibited granulocytic/monocytic differentiation but increased erythroid/megakaryocytic differentiation (Cooks et al., 1999). In this study, we quantified the level of HOXA5 mRNA by real-time RT-PCR and examined its correlation with the clinical outcome. We found HOXA5 expression was significantly up-regulated in AML compared to healthy controls, especially in non-remission patients. High HOXA5 expression was observed in 50.7\% of AML patients if the cut-off value 0.000102 was used according to ROC curve. Our study further demonstrated that high HOXA5 expression was significantly associated with CR rate of AML patients, although we did not find the correlation of HOXA5 expression with other clinical parameters. HOXA9 gene is localized on the human chromosome $7 \mathrm{p} 15$, which is relevant not only to the function of the early hemopoietic stem cells, it also gets involved at the late differentiation and orientation stages of hemopoietic stem cells (Golub et al., 1999). Some data indicated that HOXA9 gene has medullary system specificity, its expression was relevant to the immature leukemia cells, and it might play a role in the pathogenesis of AML (Thorsteinsdottir et al., 2001). Here we found HOXA9 expression was significantly upregulated in AML compared with healthy controls, especially in non-M2 and M3 AML patients. High HOXA9 expression was observed in $50.7 \%$ of AML patients if the cut-off value 0.00212 was used according to ROC curve. Lower relative amounts of HOXA9 were detected in patients with $\mathrm{t}(8 ; 21)(\mathrm{q} 22 ; \mathrm{q} 22)$ and cases with prognostically favorable cytogenetic features, but extended studies have not been able to reveal any significant associations with complete response rates of event-free survival (Drabkin et al., 2002). Our study further demonstrated that high HOXA9 expression was related with age and FAB. These findings are consistent with those of the previous study.

Regulation of HOXA5 expression requires multiple cis-acting regions, some encompassing coding sequences from neighboring genes (Boucherat et al., 2009). Our study showed that overexpression of HOXA5 closely correlated with high expression of HOXA9, which were related to the development of myelomonocytes and leukemogenesis, the correlation between HOXA5 and HOXA9 is a particularly interesting finding that calls for further research to reveal the underlying mechanism of the interaction.

In conclusion, our present study indicated that high HOXA5 expression in AML tended to increase in nonremission patients, while high expression of HOXA9 was found in patients with non-M2, M3 and AML patients with age older than 60 years, the expression of HOXA9 was low in AML patients with favorable karyotypes, such as M2 $\mathrm{t}(8 ; 21)$ (q22;q22) and M3 t (15;17) (q22;q21). 
Peng Zhao et al

Moreover, our results showed that HOXA5 correlated with a poor prognosis and HOXA9, which was correlated with leukemogenesis. However, the small sample size and the preliminary nature of this study are the limitations of our study, performing a larger study to clearly determine the relationships between HOXA5 and HOXA9, prognosis of AML patients is also needed.

\section{Acknowledgements}

The study was supported by National Natural Science Foundation of China (No. 81100379, 81472210, 81472346, 81401874, 81001212), Science and Technology Planning Project of Guangdong Province, China (No. 2013B022000102), and Medical Scientific Research Foundation of Guangdong Province, China (No. A2014292).

\section{References}

Abramovich C, Humphries RK (2005). Hox regulation of normal and leukemic hematopoietic stem cells. Curr Opin Hematol, 12, 210-16.

Arocho A, Chen B, Ladanyi M, Pan Q (2006). Validation of the 2-DeltaDeltaCt calculation as an alternate method of dataanalysis for quantitative PCR of BCR-ABL P210 transcripts. Diagn Mol Pathol, 15, 56-61.

Bennett JM, Catovsky D, Daniel MT, Flandrin G, et al (1985). Proposed revised criteria for the classification of acute myeloid leukemia. A report of the French-American-British Cooperative Group. Ann Intern Med, 103, 620-5.

Borrow J, Shearman AM, Stanton VP Jr, et al (1996). The t (7;11) (p15;p15) translocation in acute myeloid leukaemia fuses the genes for nucleoporinNUP98 and class I homeoprotein HOXA9. Nat Genet, 12, 159-67.

Boucherat O, Guillou F, Aubin J, et al (2009). Hoxa5: a master gene with multifaceted roles. Med Sci (Paris), 25, 77-82.

Cancer Genome Atlas Research Network (2013). Genomic and epigenomic landscapes of adult de novo acute myeloid leukemia. N Engl J Med, 368, 2059-74. '

Cillo C, Cantile M, Faiella A, et al (2001). Homeobox genes in normal and malignant cells. J Cell Physiol, 188, 161-9.

Cooks GM, Fuller J, Petersen D, et al (1999). Constitutive HOXA5 expression inhibits erythropoiesis and increases myelopoiesis from humanhematopoietic progenitors. Blood, 94, 519-28.

Drabkin HA, Parsy C, Ferguson K, et al (2002). Quantitative HOX expression in chromosomally defined subsets of acute myelogenousleukemia. Leukemia, 16, 186-95.

Estey E, Döhner H (2006). Acute myeloid leukaemia. Lancet, 368, 1894-90.

Fuller JF, McAdara J, Yaron Y, et al (1999). Characterization of HOX gene expression during myelopoiesis: role of HOX A5 in lineage commitment and maturation. Blood, 93, 3391-400.

Golub TR, Slonim DK, Tamayo P, et al (1999). Molecular classification of cancer: class discovery and class prediction by gene expression monitoring. Science, 286, 531-7.

Gonzalez Garcia JR, Meza-Espinoza JP (2006). Use of the International System for Human Cytogenetic Nomenclature (ISCN). Blood, 108, 3952-3.

Hou HA, Lin CC, Chou WC, et al (2014). Integration of cytogenetic and molecular alterations in risk stratification of 318 patients with denovo non-M3 acute myeloid leukemia. Leukemia, 28, 50-8.

Kumar A, Vashist M, Rathee R (2014). Maternal factors and risk of childhood leukemia. Asian Pac J Cancer Prev, 15, 781-4.

Li Y, Lin J, Yang J, Qian J, et al (2013). Overexpressed let-7a-3 is associated with poor outcome in acute myeloid leukemia. Leuk Res, 37, 1642-7.

Nakamura T, Largaespada DA, Lee MP, Johnson LA et al (1996). Fusion of the nucleoporin gene NUP98 to HOXA9 by the chromosome translocation $\mathrm{t}(7 ; 11)(\mathrm{p} 15 ; \mathrm{p} 15)$ in human myeloid leukaemia. Nat Genet, 12, 154-8.

Nakamura T, Yamazaki Y, Hatano Y, et al (1999). NUP98 is fused to PMX1 homeobox gene in human acute myelogenous leukemia withchromosome translocation $\mathrm{t}(1 ; 11)$ (q23;p15). Blood, 94, 741-7.

Sabattini E, Bacci F, Sagramoso C, et al (2008). WHO classification of tumours of haematopoietic and lymphoid tissues in 2008: an overview. Pathologica, 102, 83-8.

Shahab S, Shamsi TS, Ahmed N (2013). Prognostic involvement of nucleophosmin mutations in acute myeloid leaukemia. Asian Pac J Cancer Prev, 14, 5615-20.

Spencer DH, Young MA, Lamprecht TL, et al (2015). Epigenomic analysis of the HOX gene loci reveals mechanisms that may control canonical expression patterns in AML and normal hematopoietic cells.Leukemia. 2015 Jan 20.

Thorsteinsdottir U, Kroon E, Jerome L, et al (2001). Defining roles for HOX and MEIS1 genes in induction of acute myeloid leukemia. Mol Cell Biol, 21, 224-34.

van Oostveen J, Bijl J, Raaphorst F, et al (1999). The role of homeobox genes in normal hematopoiesis and hematological malignancies. Leukemia, 13, 1675-90. 